\title{
Monte Carlo simulation of coherent effects in multiple scattering
}

\author{
By Igor V. Meglinskiªr, Vladimir L. KuZmin², \\ Dmitry Y. Churmakov ${ }^{1}$ and Douglas A. Greenhalgh ${ }^{1}$ \\ ${ }^{1}$ School of Engineering, Cranfield University, \\ Cranfield MK43 OAL, UK (i.meglinski@cranfield.ac.uk) \\ ${ }^{2}$ St Petersburg Institute of Commerce and Economics, \\ Novorossiyskaya ul. 50, St Petersburg 194021, Russia
}

Using a combination of the stochastic Monte Carlo technique and the iteration procedure of the solution to the Bethe-Salpeter equation, it has been shown that the simulation of the optical path of a photon packet undergoing an $n$th scattering event directly corresponds to the $n$ th-order ladder diagram contribution. In this paper, the Monte Carlo technique is generalized for the simulation of the coherent backscattering and temporal correlation function of optical radiation scattered within the randomly inhomogeneous turbid medium. The results of simulation demonstrate a good agreement with the diffusing wave theory and experimental results.

\footnotetext{
Keywords: Bethe-Salpeter equation; coherent back-scattering; temporal field correlation function; diffusing-wave spectroscopy; Monte Carlo simulation
}

\section{Introduction}

The stochastic numerical Monte Carlo (MC) technique is widely used in modelling of optical radiation propagation in randomly inhomogeneous highly scattering media. Various MC algorithms in a number of applications have been developed to estimate the intensity of multiple scattered light (Iwai et al. 1995; Skipetrov \& Chesnokov 1998; Skipetrov \& Meglinskii 1998; Lenke \& Maret 2000a; Churmakov et al. 2002). The particular interest is explained by the fact that, for the multiple scattering of optical radiation, the field-interference phenomena has been attracting great attention during the last decade (Ospeck \& Fraden 1994; Boas et al. 1996; Ishii et al. 1997; Lenke \& Maret 2000a, $b$; Lenke et al. 2002). This is because, despite multiple scattering of light, typical for many colloidal and biological media, coherent effects are still observed. Coherence is one of the principal parameters of laser radiation and characterizes the degree to which the oscillating electromagnetic laser radiation maintains a near-constant phase shift. The manifestation of this is observed through the interference effects, such as the coherent back-scattering (CBS) and angular and/or temporal intensity correlation functions. The radiative transfer in randomly inhomogeneous highly scattering media, including coherence effects, is generally described in frameworks of the Bethe-Salpeter equation (Kuzmin \& Romanov 1996; van Rossum \& Nieuwenhuizen 1999), however, numerical modelling of these effects requires particular approaches. In this study, based on the combination of the MC technique and 


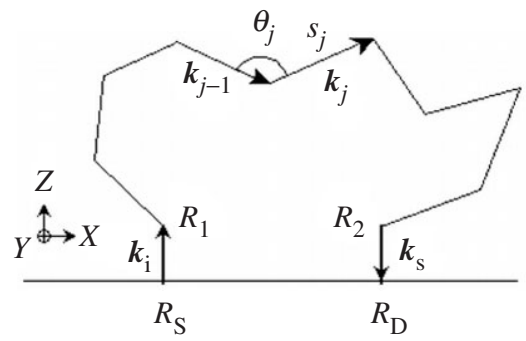

Figure 1. Schematic of MC photon packet random walk between the points of source $\boldsymbol{R}_{\mathrm{S}}$ and detector $\boldsymbol{R}_{\mathrm{D}} \cdot \boldsymbol{R}_{1}$ and $\boldsymbol{R}_{2}$ are the points of the first and final scatterings, respectively. The Cartesian coordinate system is used to specify a position of a photon packet within the medium. The vectors $\boldsymbol{k}_{j-1}$ and $\boldsymbol{k}_{j}$ are wave vectors before and after the $j$ th scattering event, $\theta_{j}$ is the angle between them and $s_{j}$ is the free path length.

the Bethe-Salpeter equation, represented as a series of the ladder diagrams, we generalize the computational technique in frame of a common approach to the CBS and temporal field correlation function calculations. We consider the normal incidence of radiation at a semi-infinite non-absorbing scattering medium with a plain boundary interface as a typical case in theoretical studies.

\section{Correlation transfer theory}

The stochastic MC method for numerical calculation of the intensity radiation diffusely scattered within a randomly inhomogeneous turbid medium is based on the consequent simulation of the photon trajectories within the medium between the points of the source $\boldsymbol{R}_{\mathrm{S}}$ and the detector $\boldsymbol{R}_{\mathrm{D}}$ (Churmakov et al. 2002). The simulation of a single trajectory consists of the following stages: injection of the photon packet in medium; generation of the photon path-length; generation of scattering events; definition of reflection/refraction at the medium boundary; definition of detection and accounting for the absorption. A typical trajectory of the photon-packet random walks is schematically shown in figure 1 .

Let a medium occupy a half-space $z>0$, where $z$ is the Cartesian coordinate normal to the boundary. Let $\boldsymbol{k}_{\mathrm{i}}$ and $\boldsymbol{k}_{\mathrm{s}}$ be the wave vectors of incident and scattered plain waves.

In the MC approach, calculation of the CBS and temporal correlations in the plane-wave geometry is simply realized by fixing the directions of the incident and emerging photon packets at the point $\boldsymbol{R}_{\mathrm{S}}$ and $\boldsymbol{R}_{\mathrm{D}}$, respectively, and subsequent integration over all possible $\boldsymbol{R}_{\mathrm{D}}$ positions.

The radiative transfer in a randomly inhomogeneous medium with temporal and spatial fluctuations of the dielectric permittivity is described by the Bethe-Salpeter equation,

$$
\begin{aligned}
\Gamma\left(\boldsymbol{R}_{2}, \boldsymbol{R}_{1}, t \mid \boldsymbol{k}_{\mathrm{s}}, \boldsymbol{k}_{\mathrm{i}}\right)= & \mu_{\mathrm{s}} p_{t}\left(\boldsymbol{k}_{\mathrm{i}}-\boldsymbol{k}_{\mathrm{s}}\right) \delta\left(\boldsymbol{R}_{2}-\boldsymbol{R}_{1}\right) \\
& +\mu_{\mathrm{s}} \int p_{t}\left(\boldsymbol{k}_{\mathrm{s}}-\boldsymbol{k}_{23}\right) \Lambda\left(R_{23}\right) \Gamma\left(\boldsymbol{R}_{3}, \boldsymbol{R}_{1}, t \mid \boldsymbol{k}_{23}, \boldsymbol{k}_{\mathrm{i}}\right) \mathrm{d} \boldsymbol{R}_{3} .
\end{aligned}
$$

Here, $\Gamma\left(\boldsymbol{R}_{2}, \boldsymbol{R}_{1}, t \mid \boldsymbol{k}_{\mathrm{s}}, \boldsymbol{k}_{\mathrm{i}}\right)$ is Green's function or propagator of the Bethe-Salpeter equation. It describes the propagation of two, delayed in time $t$, complex-conjugated 


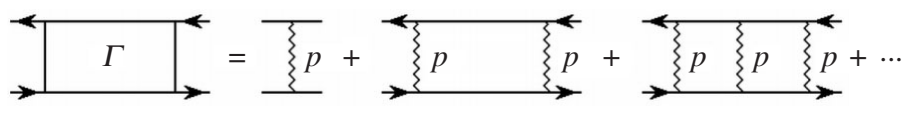

Figure 2. Schematic of an iterative solution to the Bethe-Salpeter equation as a series of ladder diagrams. $\Gamma$ is Green's function or the propagator of the Bethe-Salpeter equation and $p$ is the scattering phase function.

electromagnetic fields from $\boldsymbol{R}_{1}$ to $\boldsymbol{R}_{2}$. Vector $\boldsymbol{k}_{23}=k\left(\boldsymbol{R}_{2}-\boldsymbol{R}_{3}\right)\left|\boldsymbol{R}_{2}-\boldsymbol{R}_{3}\right|^{-1}$ is the wave vector of radiation propagating from $\boldsymbol{R}_{2}$ to $\boldsymbol{R}_{3}, k=n k_{0}$, where $k_{0}=2 \pi / \lambda$ is the wavenumber, $\lambda$ is the wavelength and $n=n_{1}+\mathrm{i} n_{2}$ is the refractive index of the medium. The real part $n_{1}$ determines the reflectivity mismatch at the medium boundary. The imaginary part of $n$ determines the photon mean free path length $\left(2 n_{2} k_{0}\right)^{-1}=l=\mu_{t}^{-1}$. Generally, the extinction coefficient $\mu_{t}$ is a sum of the scattering coefficient $\mu_{\mathrm{s}}$ and absorption coefficient $\mu_{\mathrm{a}}, \mu_{t}=\mu_{\mathrm{s}}+\mu_{\mathrm{a}}$. For a non-absorbing medium, $\mu_{t}=\mu_{\mathrm{s}}$ and $k_{\mathrm{s}}=k_{\mathrm{i}}=k$.

Propagator $\Lambda(R)=R^{-2} \exp (-R / l)$ describes the transfer of optical radiation between two successive scattering events, and stems from the product of the two complex-conjugated Green functions. The function $p_{t}\left(\boldsymbol{k}_{j-1}-\boldsymbol{k}_{j}\right)$ is defined as

$$
p_{t}\left(\boldsymbol{k}_{j-1}-\boldsymbol{k}_{j}\right)=\frac{G\left(\boldsymbol{k}_{j-1}-\boldsymbol{k}_{j}, t\right)}{\int G\left(\boldsymbol{k}_{j-1}-\boldsymbol{k}_{j}, 0\right) \mathrm{d} \Omega}
$$

and coincides with the scattering phase function at $t=0, p_{t}\left(\boldsymbol{k}_{j-1}-\boldsymbol{k}_{j}\right)$ and $\mathrm{d} \Omega$ denotes a solid angle element about the direction of wave vector. Here, $G\left(\boldsymbol{k}_{j-1}-\boldsymbol{k}_{j}, t\right)$ is the Fourier transform of the permittivity correlation function,

$$
G(\boldsymbol{q}, t)=\frac{1}{(4 \pi)^{2}} \int \mathrm{d} \boldsymbol{r}\langle\delta \varepsilon(0,0) \delta \varepsilon(\boldsymbol{r}, t)\rangle \exp (-\mathrm{i} \boldsymbol{q} \cdot \boldsymbol{r}),
$$

and, for $t=0, G(\boldsymbol{q}, 0)$ gives the cross-section of single scattering. The optical theorem relates $G(\boldsymbol{q}, 0)$ and the scattering length $l_{\mathrm{s}}=\mu_{\mathrm{s}}^{-1}$. In the context of the Born approximation, this relation yields

$$
\frac{1}{l_{\mathrm{s}}}=k_{0}^{4} \int G\left(\boldsymbol{k}_{j-1}-\boldsymbol{k}_{j}, 0\right) \mathrm{d} \Omega .
$$

Iterating the Bethe-Salpeter equation (2.1), one obtains the series in scattering orders,

$$
\begin{aligned}
& \Gamma\left(\boldsymbol{R}_{2}, \boldsymbol{R}_{1}, t \mid \boldsymbol{k}_{\mathrm{s}}, \boldsymbol{k}_{\mathrm{i}}\right) \\
& \quad=\mu_{\mathrm{s}} p_{t}\left(\boldsymbol{k}_{\mathrm{i}}-\boldsymbol{k}_{\mathrm{s}}\right) \delta\left(\boldsymbol{R}_{2}-\boldsymbol{R}_{1}\right)+\mu_{\mathrm{s}}^{2} p_{t}\left(\boldsymbol{k}_{\mathrm{s}}-\boldsymbol{k}_{21}\right) \Lambda\left(R_{21}\right) p_{t}\left(\boldsymbol{k}_{21}-\boldsymbol{k}_{\mathrm{i}}\right) \\
& \quad+\mu_{\mathrm{s}}^{3} p_{t}\left(\boldsymbol{k}_{\mathrm{s}}-\boldsymbol{k}_{23}\right) \Lambda\left(R_{23}\right) p_{t}\left(\boldsymbol{k}_{23}-\boldsymbol{k}_{31}\right) \Lambda\left(R_{31}\right) p_{t}\left(\boldsymbol{k}_{31}-\boldsymbol{k}_{\mathrm{i}}\right)+\cdots .
\end{aligned}
$$

This series is usually illustrated by ladder diagrams (figure 2).

In the far-field zone, the temporal field correlation function of scattered radiation can be expressed as the sum of the non-coherent and interference components,

$$
C_{E}\left(t \mid \boldsymbol{k}_{\mathrm{s}}, \boldsymbol{k}_{\mathrm{i}}\right)=C^{(L)}+C^{(V)} .
$$

Here, $C^{(L)}$ is the non-coherent component,

$$
C^{(L)}\left(t \mid \boldsymbol{k}_{\mathrm{s}}, \boldsymbol{k}_{\mathrm{i}}\right)=\int \mathrm{d} \boldsymbol{R}_{1} \mathrm{~d} \boldsymbol{R}_{2} \Gamma\left(\boldsymbol{R}_{1}, \boldsymbol{R}_{2}, t \mid \boldsymbol{k}_{\mathrm{s}}, \boldsymbol{k}_{\mathrm{i}}\right) \exp \left(-\frac{z_{1}}{l \cos \theta_{\mathrm{i}}}-\frac{z_{2}}{l \cos \theta_{\mathrm{s}}}\right),
$$


and $C^{(V)}$ is the interference component,

$$
\begin{aligned}
& C^{(V)}\left(t \mid \boldsymbol{k}_{\mathrm{s}}, \boldsymbol{k}_{\mathrm{i}}\right) \\
& =\int \mathrm{d} \boldsymbol{R}_{1} \mathrm{~d} \boldsymbol{R}_{2}\left[\Gamma\left(\boldsymbol{R}_{1}, \boldsymbol{R}_{2}, t \mid \frac{1}{2}\left(\boldsymbol{k}_{\mathrm{s}}-\boldsymbol{k}_{\mathrm{i}}\right), \frac{1}{2}\left(\boldsymbol{k}_{\mathrm{i}}-\boldsymbol{k}_{\mathrm{s}}\right)\right)\right. \\
& \left.\quad-k_{0}^{4} G\left(\boldsymbol{k}_{\mathrm{s}}-\boldsymbol{k}_{\mathrm{i}}, t\right) \times \delta\left(\boldsymbol{R}_{2}-\boldsymbol{R}_{1}\right)\right] \\
& \quad \times \exp \left[-\frac{z_{1}+z_{2}}{2 l}\left(\frac{1}{\cos \theta_{\mathrm{i}}}+\frac{1}{\cos \theta_{\mathrm{s}}}\right)+\mathrm{i} n_{1} k_{0}\left(z_{1}-z_{2}\right)\left(\cos \theta_{\mathrm{i}}-\cos \theta_{\mathrm{s}}\right)\right. \\
& \left.\quad+\mathrm{i} n_{1} k_{0}\left(x_{1}-x_{2}\right)\left(\sin \theta_{\mathrm{i}}-\sin \theta_{\mathrm{s}}\right)\right],
\end{aligned}
$$

where $\theta_{\mathrm{i}}$ and $\theta_{\mathrm{s}}$ are the angles of incident and outgoing photon packets, both laying in the $(x, z)$-plane (see figure 1$)$.

In case of retro-reflection, i.e. $\boldsymbol{k}_{\mathrm{s}}=-\boldsymbol{k}_{\mathrm{i}}$, the interference component $C^{(V)}\left(t \mid \boldsymbol{k}_{\mathrm{s}}, \boldsymbol{k}_{\mathrm{i}}\right)$ is equal to $C^{(L)}\left(t \mid \boldsymbol{k}_{\mathrm{s}}, \boldsymbol{k}_{\mathrm{i}}\right)$, wherein the single scattering term is excluded.

The incoherent component determines the temporal field correlation function,

$$
g_{1}(t)=\frac{C^{(L)}\left(t \mid-\boldsymbol{k}_{\mathrm{i}}, \boldsymbol{k}_{\mathrm{i}}\right)}{C^{(L)}\left(0 \mid-\boldsymbol{k}_{\mathrm{i}}, \boldsymbol{k}_{\mathrm{i}}\right)} .
$$

The intensity correlation function is obtained as a quadratic form $g_{2}(t)=1+g_{1}^{2}(t)$. Equation (2.8) at $t=0$ determines the normalized CBS peak,

$$
I_{\mathrm{CBS}}\left(\theta_{\mathrm{s}}\right)=\frac{C^{(V)}\left(0 \mid \boldsymbol{k}_{\mathrm{s}}, \boldsymbol{k}_{\mathrm{i}}\right)}{C^{(V)}\left(0 \mid-\boldsymbol{k}_{\mathrm{i}}, \boldsymbol{k}_{\mathrm{i}}\right)},
$$

describing its angular dependence.

\section{Comparative analysis of MC simulation and scattering order series approach}

Let us compare an analytic procedure of summing up the ladder-diagram series with the MC technique for calculation of the intensity of radiation scattered within a non-absorbing $\left(l=l_{\mathrm{s}}\right)$ turbid medium at the stationary conditions. The first term of the iterative series describes the single scattering, the second term describes two scattering events, etc. Similarly, the MC method describes the radiation propagation as a stochastic process consisting of $1,2,3 \ldots, N$ scattering events. The addition of extra ladder section $\Lambda\left(R_{j-1 j}\right) p_{0}\left(\boldsymbol{k}_{j-1}-\boldsymbol{k}_{j}\right)$ in the theoretical description is realized in the numerical experiment by simulating a photon, which travels a certain distance $s$ to the next scattering event.

In the MC method, the free photon path $s$ between two successive scattering events is governed by the Poisson distribution (Sobol' 1974),

$$
f(s)=\mu_{t} \exp \left(-\mu_{t} s\right) .
$$

The probability $\xi$ that the photon travels a path exceeding $s$ is equal to

$$
\xi=\int_{\mathrm{s}}^{\infty} f\left(s^{\prime}\right) \mathrm{d} s^{\prime} .
$$


Using the probability density function (3.1), one easily finds

$$
s=-\frac{\ln \xi}{\mu_{t}},
$$

where $\xi$ is a random number uniformly distributed in the interval $[0,1]$.

A new direction of the photon packet is determined by the scattering phase function. The photon packets are detected if they arrive at the detector with an exit position $\boldsymbol{R}_{\mathrm{D}}$ and angle lying within the detection conditions. Then a sequent scattering event is simulated by repeating the steps described above.

Physically, a series arising upon the iteration of (2.1) is an expansion in orders of scattering. If the integration over $R_{\mathrm{i}}$ is replaced by a random choice $R_{\mathrm{i}}=s$ and the integration over directions of $\boldsymbol{R}_{\mathrm{i}}$ is realized by a random choice of angles with the statistical weight determined by the phase function, then the solution to the Bethe-Salpeter equation is simulated by the MC scheme described above.

Due to the fact that the phase function is normalized to unity,

$$
\int p_{0}\left(\boldsymbol{k}_{j-1}-\boldsymbol{k}_{j}\right) \mathrm{d} \Omega=1,
$$

the statistical weight of the photon packet within the MC technique does not change during the scattering. In the theoretical approach, the statistical weight of the photon packet is conserved due to the optical theorem. Indeed, due to the identity $\int \Lambda(R) \mathrm{d} \boldsymbol{R}=4 \pi l$, the expansion parameter of an iterative series (2.5) can be presented as

$$
\mu_{\mathrm{s}} \int \mathrm{d} \boldsymbol{R}_{j} \Lambda\left(R_{j-1 j}\right) p_{0}\left(\boldsymbol{k}_{j-1}-\boldsymbol{k}_{j}\right)=\mu_{\mathrm{s}} l
$$

which turns to unity in a non-absorbing medium. The Henyey-Greenstein phase function is used to describe an anisotropic scattering (Henyey \& Greenstein 1941). A consideration of light scattering within an absorbing medium $\left(\mu_{\mathrm{s}} l \neq 1\right)$ requires a proportional reduction of the statistical weight of photon packet by a factor,

$$
\mu_{\mathrm{s}} l=\left(1+l_{\mathrm{s}} / l_{\mathrm{a}}\right)^{-1},
$$

where $l_{\mathrm{a}}=\mu_{\mathrm{a}}^{-1}$ is the absorption length. Details on the reflection/refraction at the medium boundary are given elsewhere and not considered in current paper (Churmakov et al. 2002).

\section{Calculation of the CBS and the temporal correlation function}

Based on a comparison of the MC method and the analytical series (2.5), it is possible to generalize the $\mathrm{MC}$ technique to accomplish simulation of the CBS and temporal field correlation function of the optical radiation scattered within the randomly inhomogeneous turbid medium.

Calculation of the temporal intensity correlation function is similar to the spatially resolved reflectance simulation (Churmakov et al. 2002). The only difference is that, after each scattering event, the statistical weight of a photon packet is multiplied by the phase function $p_{t}\left(\boldsymbol{k}_{j-1}-\boldsymbol{k}_{j}\right)$.

Diffusing-wave spectroscopy (DWS) (Maret \& Wolf 1987; Pine et al. 1988) studies temporal evolution of inhomogeneities within a medium. In most applications, one 
considers the Brownian mechanism of temporal decay of inhomogeneity fluctuations presenting the temporal permittivity correlation function as a product of the static correlator $G(q, 0)$ and exponential function

$$
G(q, t) \approx G(q, 0) \exp \left(-D_{\mathrm{s}} q^{2} t\right) .
$$

Here, $D_{\mathrm{s}}$ is the self-diffusion coefficient and $q$ is a wave vector transfer. Thus, in the $\mathrm{MC}$ technique, proceeding from the calculation of the intensity to the calculation of the temporal correlation function, one has to replace the phase function according to

$$
p_{0}\left(\boldsymbol{k}_{j-1}-\boldsymbol{k}_{j}\right) \rightarrow p_{0}\left(\boldsymbol{k}_{j-1}-\boldsymbol{k}_{j}\right) \exp \left(-D_{\mathrm{s}}\left|\boldsymbol{k}_{j-1}-\boldsymbol{k}_{j}\right|^{2} t\right) .
$$

In the diffusion approximation, the momentum transfer is changed to its average,

$$
\exp \left(-D_{\mathrm{s}}\left|\boldsymbol{k}_{j-1}-\boldsymbol{k}_{j}\right|^{2} t\right) \rightarrow \exp \left(-\frac{1}{2}\left(t / \tau_{0}\right)\left(l / l^{*}\right)\right),
$$

where $\tau=\left(4 D_{\mathrm{s}} k^{2}\right)^{-1}$ is the characteristic time of scattering particle diffusion at a distance comparable with the wavelength, $l^{*}=l_{\mathrm{S}}(1-\langle\cos \theta\rangle)^{-1}$ is the transport length and

$$
\langle\cos \theta\rangle=\frac{\int \mathrm{d} \Omega p\left(\boldsymbol{k}_{j-1}-\boldsymbol{k}_{j}\right) \cos \theta_{j}}{\int \mathrm{d} \Omega p\left(\boldsymbol{k}_{j-1}-\boldsymbol{k}_{j}\right)}
$$

is the average cosine of a scattering angle, describing the anisotropy of the phase function. In case of strong anisotropy, $1-\langle\cos \theta\rangle \ll 1$, the factor $\left(t / \tau_{0}\right)\left(l / l^{*}\right)$ is still small enough, even if $t / \tau_{0} \sim 1$.

The MC simulation of the CBS peak can also be realized in a similar manner. First, one has to exclude the single scattering from the detected signal, as it does not contribute to the interference term. In case of normal incidence and a small backscattering angle $\theta_{\mathrm{s}}$, one can put $\frac{1}{2}\left(\boldsymbol{k}_{\mathrm{i}}-\boldsymbol{k}_{\mathrm{s}}\right)=\boldsymbol{k}_{\mathrm{i}}$ and $\cos \theta_{\mathrm{s}} \approx \cos \theta_{\mathrm{i}}=1$ in (2.8). Due to the translational invariance with respect to the $(x, y)$-plane, we replace the exponential factor $\exp \left(\mathrm{i} \boldsymbol{q}_{\perp}\left(\boldsymbol{R}_{\mathrm{S}}-\boldsymbol{R}_{\mathrm{D}}\right)_{\perp}\right)$ with $\cos \left(\boldsymbol{q}_{\perp}\left(\boldsymbol{R}_{\mathrm{S}}-\boldsymbol{R}_{\mathrm{D}}\right)_{\perp}\right)$. Here, the subscript ' $\perp$ ' denotes the component of a vector transversal to the normal.

Thus, in order to simulate the CBS peak, one calculates a product of the weight of a photon packet detected on the surface at the distance $\left|\left(\boldsymbol{R}_{\mathrm{S}}-\boldsymbol{R}_{\mathrm{D}}\right)_{\perp}\right|$ from the point of incidence and the factor $\cos \left(\boldsymbol{q}_{\perp}\left(\boldsymbol{R}_{\mathrm{S}}-\boldsymbol{R}_{\mathrm{D}}\right)_{\perp}\right)$.

All the photon packets are launched normally to and collected over the boundary surface of the medium. No refractive index mismatch is assumed at the boundary $n_{\text {medium }}=n_{\text {ambient medium }}=1$. Photons are detected in a solid angle defined by the numerical aperture of the detector. The latter is taken as $90^{\circ}$ and $1^{\circ}$ for the temporal correlation function and CBS calculations, respectively. The MC procedure takes approximately one hour to simulate $10^{5}$ detected photons in calculations on a $1.8 \mathrm{GHz}$ Pentium 4 CPU.

\section{Results and discussion}

The results of calculation of the temporal field correlation functions for scattering slabs of thickness $L$ with different anisotropy $(\langle\cos \theta\rangle=0,0.5$ and 0.9$)$ are presented in figures 3 and 4 . The optical properties of slabs are $\mu_{\mathrm{s}}=30 \mathrm{~mm}^{-1}$ and $\mu_{\mathrm{a}}=0$. Thus the transport mean free path lengths are $l^{*}=33.3,66.6$ and $333.3 \mu \mathrm{m}$ for isotropic $(\langle\cos \theta\rangle=0)$, intermediate $(\langle\cos \theta\rangle=0.5)$ and high-anisotropy $(\langle\cos \theta\rangle=0.9)$ scattering media, respectively. The thickness of slabs $L$ is selected based on the value of transport path length $l^{*}$, as $L=m l^{*}$, with $m=1,2,5,10$. 


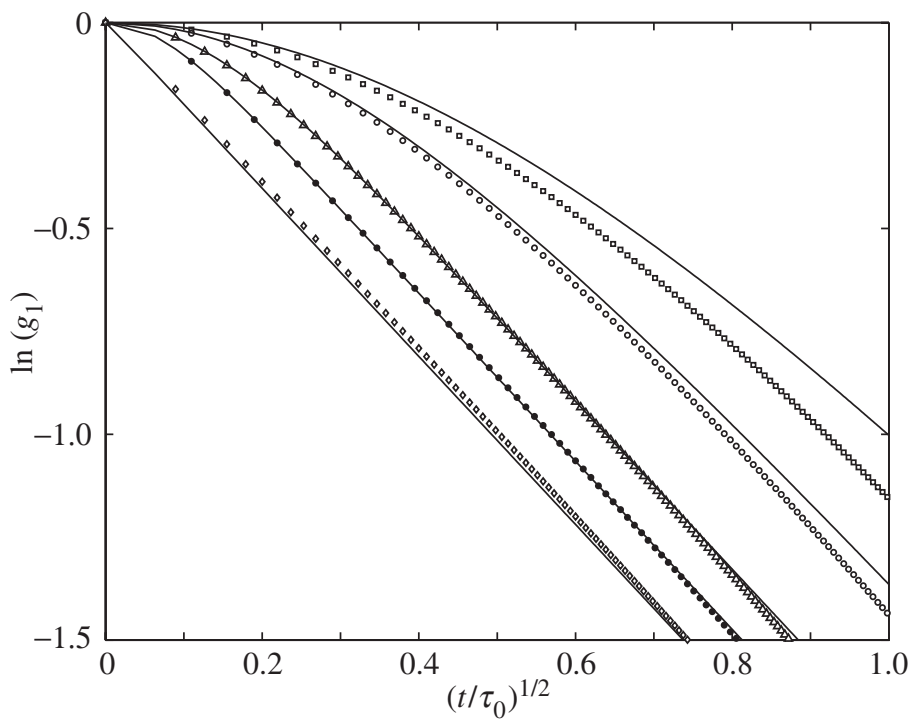

Figure 3. The normalized temporal field correlation functions $\ln \left(g_{1}\right)$ versus $\left(t / \tau_{0}\right)^{1 / 2}$ for the factor of anisotropy $\langle\cos \theta\rangle=0.5$. Symbols correspond to different slab thicknesses: $\square, L=l^{*}$; ○, $L=2 l^{*} ; \Delta, L=5 l^{*} ; \bullet, L=10 l^{*} ; \diamond$, semi-infinite medium. Solid lines show the diffusion theory prediction for a non-absorbing slab (equation (5.1)).

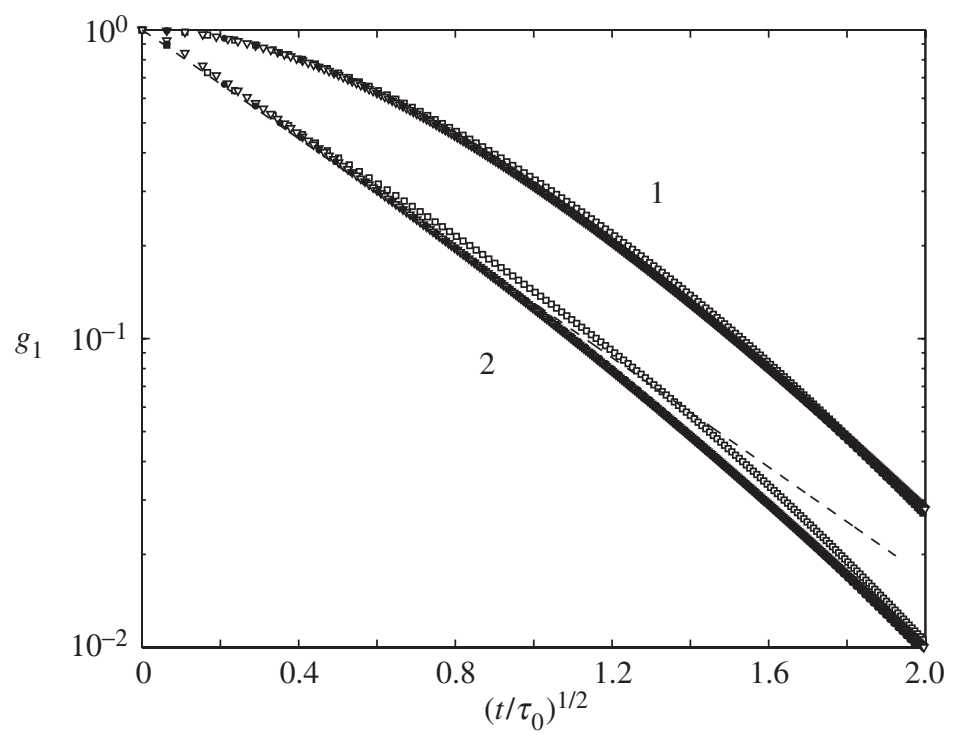

Figure 4. The normalized temporal field correlation functions $g_{1}(t)$ for semi-infinite medium versus $\left(t / \tau_{0}\right)^{1 / 2}$ for different factors of anisotropy: $\square$, isotropic $(\langle\cos \theta\rangle=0)$; •, intermediate $(\langle\cos \theta\rangle=0.5) ; \Delta$, high anisotropic $(\langle\cos \theta\rangle=0.9)$. the dashed line is the DWS prediction.

The results of simulation of the temporal field correlation function $g_{1}(t)$ versus $\left(t / \tau_{0}\right)^{1 / 2}$ for the set of slabs $L$ are presented in figure 3 . The parameter $\tau_{0}$ is taken as $0.933 \mathrm{~ms}$ (Skipetrov \& Meglinskii 1998; Skipetrov \& Chesnokov 1998). 


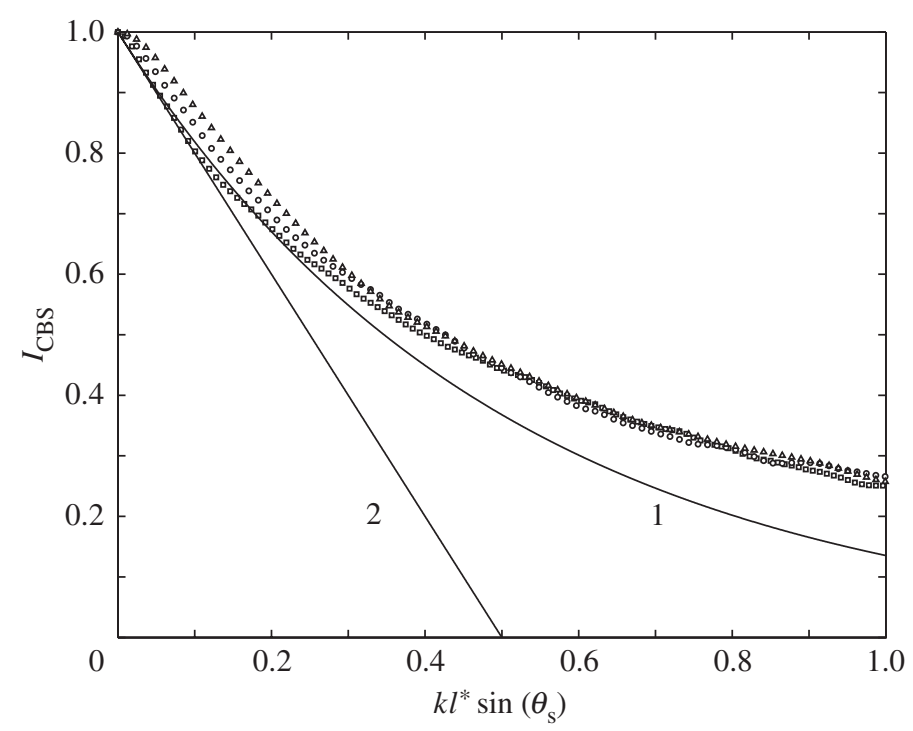

Figure 5. The peak of CBS $\left(I_{\mathrm{CBS}}\right)$ as a function of $k l^{*} \sin \theta_{\mathrm{s}}: \square,\langle\cos \theta\rangle=0 ; \circ,\langle\cos \theta\rangle=0.5$; $\boldsymbol{\Delta},\langle\cos \theta\rangle=0.9$. Solid lines represent (1) equation (5.2) and (2) equation (5.3).

The obtained results (see figure 3) are reasonably well described by the analytical formula (Pine et al. 1990)

$$
g_{1}(\tau) \propto \frac{\sinh \left(\alpha\left[L+z_{1}-z_{0}\right]\right)}{\sinh \left(\alpha\left[L+2 z_{1}\right]\right)},
$$

where $\alpha=\left(3 t /\left(\tau_{0} l^{* 2}\right)\right)^{1 / 2}, z_{0}=l^{*}$ is the depth at which the incident light is completely diffused and $z_{1} \approx \frac{2}{3} l^{*}$ is the extrapolated boundary length. Since the influence of the factor of anisotropy decreases as a slab becomes thicker, there is a tendency for a better agreement between the results of the MC simulation and that of the diffusion theory (see figure 3). We ascribe that to the diminishing role of lower-order scatterings, which cannot be properly accounted for within the diffusion theory.

The results for a semi-infinite medium (figure 4) are in a good agreement with the predictions of the DWS theory (Pine et al. 1988; MacKintosh \& John 1989). These results exhibit quite weak dependence of the temporal correlation function on the anisotropy. However, for large values of $t / \tau_{0}$, a deviation of a logarithmic plot of $g_{1}$ from a linear dependence is observed (see figure 4). Similar behaviour of $g_{1}(t)$ was predicted earlier (Kuzmin \& Romanov 1997).

The results of the CBS peak simulation, considered for the same isotropic, intermediate and high-anisotropy semi-infinite scattering media, are presented in figure 5 .

Expressed in terms of the dimensionless parameter $\tilde{q}=k l^{*} \sin \theta_{\mathrm{s}}$, the simulated angular dependence of the CBS exposes typical behaviour, indicating that it is practically independent of anisotropy (see figure 5). The results of simulation are compared with those predicted by the analytical approach (MacKintosh \& John 1989) (see figure 5). The latter can be represented as

$$
I_{\mathrm{CBS}}^{(1)}=\exp \left(-\gamma k l^{*} \sin \theta_{\mathrm{s}}\right)
$$




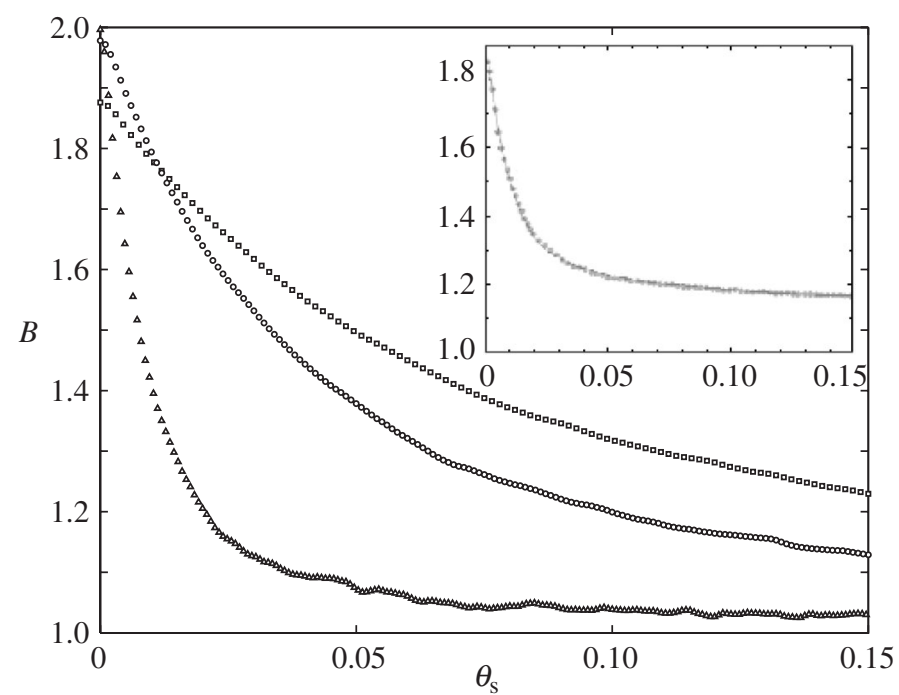

Figure 6. The angular dependence of the CBS enhancement $B$ for the different anisotropy parameter: $\square,\langle\cos \theta\rangle=0 ; \circ,\langle\cos \theta\rangle=0.5 ; \boldsymbol{\Lambda},\langle\cos \theta\rangle=0.9$. The maximum $B$ values are 1.87 , 1.97, 1.99 for $\langle\cos \theta\rangle=0,0.5$ and 0.9 , respectively. The flat part of the plot for $\langle\cos \theta\rangle=0.9$ is a bit lower then that presented by Lenke et al. (2002), due to different values of the transport path lengths: $l^{*}=333.3 \mu \mathrm{m}$ in the present simulation and $l^{*}=314 \mu \mathrm{m}$ (see inset, extracted from Lenke et al. (2002)).

or, in its linear approximation,

$$
I_{\mathrm{CBS}}^{(2)}=1-\gamma k l^{*} \sin \theta_{\mathrm{s}},
$$

where $\gamma$ is the relative slope of the CBS decay.

The results obtained are quite closely related to (5.2), wherein $\gamma=2$ (see figure 5). The results of simulation also indicate a universal decreasing trend, whereas the diffusion approximation predicts that the CBS peak falls down with different slope values for different anisotropy. The diffusion approximation (Akkermans et al. 1988),

$$
I_{\mathrm{CBS}}^{(\mathrm{diff})} \propto 1-2 \frac{\left(1+z^{*}\right)^{2}}{1+2 z^{*}} k l^{*} \sin \theta_{\mathrm{s}},
$$

yields the slope $\gamma^{(\text {diff })} \approx 2.3$ for $\langle\cos \theta\rangle=0$ and $\gamma^{(\text {diff })} \approx 0.71$ for $\langle\cos \theta\rangle \rightarrow 1$, where $z^{*}=0.71(1-\langle\cos \theta\rangle)^{-1}$.

Due to the non-zero single scattering, the CBS peak is not equal to 2. Qualitatively, this effect is apparent in figure 6, where the results of the angular dependence of the CBS intensity enhancement are presented.

The CBS intensity enhancement or parameter $B$ (see figure 6) is determined as

$$
B\left(\theta_{\mathrm{s}}\right)=1+\frac{C^{(V)}\left(0 \mid \boldsymbol{k}_{\mathrm{s}}, \boldsymbol{k}_{\mathrm{i}}\right)}{C^{(L)}\left(0 \mid-\boldsymbol{k}_{\mathrm{i}}, \boldsymbol{k}_{\mathrm{i}}\right)} .
$$

The results of MC simulation for isotropic scattering show that $B=B(0)=1.87$. It agrees with the results derived from a generalized solution of the Milne problem: $B=1.88$ (van Rossum \& Nieuwenhuizen 1999). In the limit of $\langle\cos \theta\rangle \rightarrow 1$, the 
theory predicts that $B=2$. This also agrees with the results of our MC simulation, $B \approx 1.99$ for $\langle\cos \theta\rangle=0.9$. The results of our simulations also agree with the results reported by Lenke et al. (2002).

\section{Summary}

Based on the combination of the MC technique and the solution to the BetheSalpeter equation, it has been shown that it is possible to employ a unified approach describing the coherence effects in randomly inhomogeneous disperse multiple scattering media. The results of simulation demonstrate a good agreement with the DWS theory. They are also in good agreement with the experimental results (Pine et al. 1988; MacKintosh \& John 1989) and the results of an alternative simulation (Lenke et al. 2002). Potential applications of this modelling technique are various studies of suspensions, liquid crystals, biological tissues, etc.

The authors acknowledge the support of The Royal Society (Project no. 15298) and NATO (Project PST.CLG.979652). V.L.K. acknowledges support of The Russian Foundation for Basic Research (Project no. 02-02-16577).

\section{References}

Akkermans, E., Wolf, P. E., Maynard, R. \& Maret, G. 1988 Theoretical study of the coherent backscattering of light by disordered media. J. Phys. Paris 49, 77-98.

Boas, D. A., Meglinsky, I. V., Zemany, L., Campbell, L. E., Chance, B. \& Yodh, A. G. 1996 Diffusion of temporal field correlation with selected applications. In CIS selected papers: coherencedomain methods in biomedical optics (ed. V. V. Tuchin), vol. 2732, pp. 34-46, Chicago, IL: SPIE.

Churmakov, D. Y., Meglinski, I. V. \& Greenhalgh, D. A. 2002 Influence of refractive index matching on the photon diffuse reflectance. Phys. Med. Biol. 47, 4271-4285.

Henyey, L. G. \& Greenstein, J. L. 1941 Diffuse radiation in the galaxy. Astrophys. J. 93, 70-83.

Ishii, K., Iwai, T. \& Asakura, T. 1997 Polarization properties of the enhanced backscattering of light from the fractal aggregate of particles. Opt. Rev. 4, 643-647.

Iwai, T., Furukawa, H. \& Asakura, T. 1995 Numerical analysis on enhanced back-scatterings of light based on Rayleigh-Debye scattering theory. Opt. Rev. 2, 413-419.

Kuzmin, V. L. \& Romanov, V. P. 1996 Coherent phenomena in light scattering from disordered systems. Usp. Fiz. Nauk 39, 231-260.

Kuzmin, V. L. \& Romanov, V. P. 1997 Multiply scattered light correlations in an expanded temporal range. Phys. Rev. E 56, 6008-6019.

Lenke, R. \& Maret, G. 2000a Multiple scattering of light: coherent backscattering and transmission. In Scattering in polymeric and colloidal systems (ed. W. Brown \& K. Mortensen), pp. 1-73, London: Gordon and Breach.

Lenke, R. \& Maret, G. $2000 b$ Magnetic field effects on coherent backscattering of light. Eur. Phys. J. B 17, 171-185.

Lenke, R., Tweer, R. \& Maret, G. 2002 Coherent backscattering of turbid samples containing large Mie spheres. J. Opt. A 4, 293-298.

MacKintosh, F. C. \& John, S. 1989 Diffusing wave spectroscopy and multiple scattering of light in correlated randim media. Phys. Rev. B 40, 2383-2406.

Maret, G. \& Wolf, P. E. 1987 Effect of Brownian-motion of scatters. Z. Phys. B 65, 409-413.

Ospeck, M. \& Fraden, S. 1994 Influence of reflecting boundaries and finite interfacial thickness on the coherent back-scattering cone. Phys. Rev. E 49, 4578-4589. 
Pine, D. J., Weitz, D. A., Chaikin, P. M. \& Herbolzheimer, E. 1988 Diffusing-wave spectroscopy. Phys. Rev. Lett. 60, 1134-1137.

Pine, D. J., Weitz, D. A., Zhu, J. X. \& Herbolzheimer, E. 1990 Diffusing wave spectroscopy: dynamic light scattering in the multiple scattering limit. J. Phys. Paris 51, 2101-2127.

Skipetrov, S. E. \& Chesnokov, S. S. 1998 Analysis, by the Monte Carlo method, of the validity of the diffusion approximation in a study of dynamic multiple scattering of light in randomly inhomogeneous media. Quant. Electron. 25, 753-757.

Skipetrov, S. E. \& Meglinskii, I. V. 1998 Diffusing-wave spectroscopy in random inhomogeneous media with spatially localized scatterer flow. J. Exp. Theor. Phys. 86, 661-665.

Sobol', I. M. 1974 The Monte Carlo method. University of Chicago Press.

van Rossum, M. C. W. \& Nieuwenhuizen, Th. M. 1999 Multiple scattering of classical waves: microscopy, mesoscopy and diffusion. Rev. Mod. Phys. 71, 313-371. 